\title{
DEVELOPMENT OF AN INTEGRATED SCREENER FOR UNDERNUTRITION WITHIN A COMPREHENSIVE GERIATRIC ASSESSMENT SYSTEM
}

\author{
O.R.L. Wright ${ }^{1}$, K. Klein ${ }^{2}$, P. Lakhan ${ }^{3}$, A.P. Vivanti , L.C. Gray ${ }^{5}$
}

\begin{abstract}
Objective: To develop a screener for the presence of undernutrition in older adults in acute care utilizing items within a comprehensive geriatric assessment (CGA) instrument (the interRAI Acute Care). Design: Prospective cohort study and retrospective medical record review of nutritional assessment data. Setting: Acute care tertiary teaching hospital in Brisbane, Australia. Participants: Five hundred fifty-seven general medical patients aged 70 and older admitted to the hospital. Measurements: Prevalence of geriatric syndromes at admission; measures of functional status (activities of daily living), cognition, behavioural symptoms, social support, community assistance services, health conditions, medications and other medical treatments, weight, body mass index (BMI), mode of nutritional intake; demographic variables and Subjective Global Assessment (SGA) of nutritional status. These measures were tested for their prediction of undernutrition using a logistic regression model and decision tree analysis. Results: The following variables were significant independent predictors of undernutrition on admission, after adjustment for age and gender: (i) feeling sad/depressed (OR: 3.494 [1.124-10.864]; p<0.05); (ii) short term memory recalling ability (OR: 3.325 [1.152-9.594]; $\mathrm{p}<0.05$ ); (iii) weight loss of 5\% or more in the last 30 days or $10 \%$ or more in the last 180 days (OR: 2.877 [0.9838.416]; $\mathrm{p}=0.05$ ); (iv) fatigue (OR: 3.494 [1.414-43.205]; $\mathrm{p}<0.05$ ). Decision tree analysis revealed two models most predictive of undernutrition: (i) short term memory recalling ability and depression (AUC 72.8\% [95\% CI: 65\%-80.6\%]); (ii) short term memory recalling ability and recent weight loss (5\% or more in the last 30 days or $10 \%$ or more in the last 180 days) (AUC: $74.8 \%$ [95\% CI: $65.9 \%-83.6 \%]$ ). Conclusion: Several measures within the interRAI-AC may be used as part of a screener for undernutrition in acute hospital patients aged 70 years or older. The combination of short term memory recalling ability and percentage weight loss provides the most statistically robust screener for undernutrition within the interRAI-AC.
\end{abstract}

Key words: Undernutrition, comprehensive geriatric assessment.

\section{Background}

Population aging together with increased life expectancy is resulting in inexorable growth in demand for hospital services by frail older people. In the face of finite hospital resources, there is a need to provide care efficiently, while at the same time meeting the increasing (and appropriate) expectation that care will be of the highest standard. Comprehensive geriatric assessment (CGA), and the processes embodied within it are

1. PhD, Centre for Dietetics Research (C-DIET-R), School of Human Movement and Nutrition Sciences, The University of Queensland; Brisbane, Australia; 2. PhD, Statistics Unit, QIMR Berghofer Medical Research Institute, Brisbane, Australia; Clinical Trials and Biostatistics Unit, QIMR Berghofer Medical Research Institute, Brisbane, Australia; 3. PhD, Research Fellow, Centre for Research in Geriatric Medicine, The University of Queensland, Brisbane, Australia; 4. Professional Doctorate, Research Dietitian, Department of Nutrition and Dietetics, The Princess Alexandra Hospital, Brisbane, Australia; 5. MD, PhD , Professor, Centre for Research in Geriatric Medicine, The University of Queensland, Brisbane, Australia

Corresponding Author: Dr Olivia Wright, Centre for Dietetics Research, School of Human Movement and Nutrition Sciences, The University of Queensland, Brisbane QLD Australia, Ph: +61 73365 6669; Fax: +61 73365 6877; Email: o.wright@uq.edu. au associated with more accurate diagnosis, better targeted and appropriate medical treatment, improvements in patient functional status, quality of life, disease prognosis and clinical outcomes (1). The coordinated multidisciplinary assessment process underpinning CGA facilitates rapid investigation of a range of complex, interactive co-morbidities, which can then be treated in the context of the "whole person". Efficacy of the multidisciplinary elements of CGA has been examined briefly (2); however, the validity of the measures used within CGA to assess disciplinary components needs further investigation.

The screening of nutritional status in order to identify patients who are under nourished is an important aspect of CGA, as treatment has significant potential to improve patient quality of life, clinical outcomes and reduce hospital costs (3). Undernutrition is documented in around $50-60 \%$ of acute geriatric inpatients aged 65 years or older and is recognized as a marker of frailty and prognosis (4). There are numerous tools for screening 
and assessing undernutrition which incorporate various subjective, anthropometric, clinical or biochemical components. Implementation and validation of these tools, internationally, is challenged by the absence of an absolute gold standard for nutrition assessment, time constraints, or lack of awareness of the most appropriate tools to use. Biochemical assessment methods (for example, the commonly used serum albumin) may be affected by hydration and disease processes (particularly renal and liver conditions), and thus represent client illness rather than nutrient intake (5). Therefore, studies utilizing these assessment techniques, on the whole, are more likely to obtain higher prevalence rates of undernutrition than other methods. A range of biochemical and anthropometric parameters (weight, height, body mass index [BMI], percentage weight loss, albumin) have been used as part of CGA to assess nutritional status; however, structured nutritional assessment using validated tools is not usually performed. Although numerous studies of nutrition screening and assessment tools exist, no studies assessing the agreement between nutritional measures within CGA and validated nutrition assessment tools have been completed.

Valid and reliable tools to assess nutritional status are the Subjective Global Assessment (SGA) (6) and its derivative, the Patient Generated Subjective Global Assessment (PG-SGA) (7). The SGA and PG-SGA involve assessments of medical history, a physical examination and evaluation of biochemical laboratory results (8). A combination of these items determines whether patients are classified as well nourished (Score A), mild to moderately malnourished (Score B) or severely malnourished (Score C) (6). Nutritional status (A, B or $C$ ) is documented in the patient's hospital medical chart. The SGA is particularly valuable for identifying those with established undernutrition in this setting (9). It demonstrates acceptable predictive ability with sensitivity and specificity of $82 \%$ and $72 \%$ respectively (6).

This paper presents a sub-analysis of data from a larger cohort study examining the prevalence of geriatric syndromes and outcomes in an acute hospital setting (10). The purpose of this sub-analysis was to determine which geriatric syndromes or related variables within a CGA instrument used internationally were significantly associated with the presence of undernutrition on admission to acute care and could therefore be used to derive a new screener for undernutrition within CGA.

\section{Methods}

\section{Study sample}

Patients admitted to the General Medical Unit (GMU) of a large tertiary teaching hospital in Brisbane, Australia
(2005-2008), aged $\geq 70$ years, who were expected to remain in hospital for more than 48 hours were eligible for inclusion in this study. Consent was obtained from patients on admission to the GMU. Exclusion criteria included: (i) patients admitted to intensive or coronary care units; (ii) patients admitted to the general medical unit for terminal care only, and (iii) patients transferred from a general medical to another unit within 24 hours of admission. When cognitive impairment was documented in the medical notes or suspected by a study assessor (in the absence of any medical documentation), consent from the relevant proxy was obtained. Demographic data were obtained from the medical record system in relation to non-consenting patients to establish comparability to consenting patients.

\section{Data collection instruments and methods}

The interRAI-Acute Care (interRAI-AC) was used for comprehensive geriatric assessment in this study (11) and includes data pertaining to geriatric syndromes (and risks of acquiring them) collected in the pre-morbid, admission and discharge periods. The interRAI-AC provides a comprehensive evaluation of older patients' functional status, geriatric syndromes, cognition, behavioural symptoms, social supports, community assistance services, health conditions, medications and other medical treatments, weight, height, percentage weight loss over time and mode of nutritional intake, and all of these domains were examined in this study. The methods and reliability of data collection for all of these domains is detailed elsewhere $(12,13)$. Admission data was used for the purposes of this study and trained nurses conducted all assessments. Application of the interRAI-AC results in the identification and documentation of a comprehensive range of geriatric syndromes and risks, in contrast to the current paucity of documentation of these parameters in patient medical files noted in the literature (14).

A retrospective medical record review was completed for this study to obtain SGA data that matched the patients included from 2005-2008, identified by hospital record number and date of birth. Exclusion criteria for this routine data collection included patients with acute psychiatric conditions. The SGAs had been completed by hospital dietitians fully trained in its administration, as part of standard clinical practice. Standard practice at the hospital involved using the Malnutrition Screening Tool (MST) to identify patients at risk of malnutrition who required nutritional assessment using the SGA (13). A number of studies have used the SGA in older adults to assess undernutrition and have shown it is a valid, reproducible indicator of nutritional status in this group (6). Data for patients transferred from coronary or intensive care units were not available as these patients were excluded from the original cohort study where data for this study was sourced. If multiple SGAs were located throughout the hospital admission, the assessment 
completed on admission was used.

\section{Statistical analysis}

A combined version of SGA B + SGA C was used as the "undernourished" category. Continuous and normally distributed variables (age) were summarized as means with standard deviations (SD). Differences in results for all interRAI-AC measures were compared between males and females using independent t-tests for means, Chi square tests for variables with two categories and Kruskal-Wallis tests for variables with more than two categories. Statistically significant results are presented. Chi square tests were completed to test associations between clinical (geriatric) characteristics and demographic/service provision variables measured by the interRAI-AC and nutritional status defined by SGA. Items with statistically significant associations were included in backward, stepwise logistic regression models to determine independent effects. Standard regression diagnostics were performed. All models were adjusted for potential confounders (age and gender). Significant independent variables from the regression analysis were passed onto the decision tree analyses. Receiver operating characteristic (ROC) curve analyses using the results from the decision tree analyses were then performed to determine the most reliable predictors from the parsimonious model. Statistical significance was assessed at the 5\% level for all analyses. The analyses were completed using SPSS version 16.0 (SPSS Inc. Chicago, IL, USA) and $\mathrm{R}$ version 2.15.3. The Human Research Ethics Committee at the Princess Alexandra Hospital $(2004 / 079 ; 2008 / 130)$ and the Medical Research Ethics Committee at the University of Queensland (2008001564) approved all study procedures.

\section{Results}

\section{Sample characteristics}

This study included 557 patients aged 70 to 102 years (mean $82 \pm 7.1) ; 43.7 \%$ were male $(n=244 / 557)$, and 212 $(38.0 \%)$ were married. Characteristics of the sample are presented in Table 1. The median length of stay was 7 days ( 2 - 99 days). Two hundred and one patients $(36.0 \%)$ lived alone, $204(36.6 \%)$ had been admitted to hospital in the previous 90 days and $63(11.3 \%)$ were admitted from a low- or high-level long-term care facility. Forty-three per cent used formal community services before hospital admission. The most frequent admission diagnoses were falls (9\%), chest pain (5\%), urosepsis or urinary tract infection $(4 \%)$, fractured neck of femur (3\%), congestive cardiac failure $(2 \%)$ and abdominal pain $(0.9 \%)$. An additional 249 eligible patients (or their next of kin) refused consent, mainly due to ill health or not wishing to participate in research. The median age and gender distribution of excluded patients was similar to those participants in the study.

Of the 557 patients for whom interRAI-AC data was available, 111 nutritional assessments using the SGA had been completed (20\%) as part of standard dietetics practice at the hospital, and this determined the sample size available for reliability, regression and decision tree analyses. There were no statistically significant differences in characteristics including age, gender, marital status, country of birth, living arrangement, residence/transition status, time since last hospital stay, BMI or length of stay, between patients who had SGA's completed and those who did not. Females were statistically significantly older than males in the sample by around three years $(\mathrm{p}<0.001)$. Undernutrition according to the SGA was more prevalent in males than females ( $p=0.041$ ). Males were more likely to have had recent weight loss than females $(p=0.002)$, which coincides with their higher rates of undernutrition. Females were less likely to be walking independently on admission $(\mathrm{p}=0.018)$ and had higher rates of pain frequency compared to males $(p=0.002)$. Males were more independent with eating on admission than females $(p=0.034)$; however, their rates of undernutrition were higher than females $(p=0.041)$. Females were less independent than males with personal hygiene $(\mathrm{p}=0.009)$ and bathing $(\mathrm{p}=0.004)$ on admission. A significant proportion of females were widowed compared to males $(\mathrm{p}=0.001)$ and lived alone $(\mathrm{p}=0.001)$. Females appeared to utilise fewer community services prior to admission due to a higher proportion living in the institutional setting; however, further analysis indicated females were more likely to have housekeeping services $(p=0.012)$, medication aid services $(\mathrm{p}=0.019)$ and personal care assistance $(p=0.022)$. Interestingly, there was no significant difference in length of stay between those who were classified as well nourished or malnourished according to the SGA in this study $(\mathrm{p}=0.99)$.

\section{Associations between other CGA measures and undernutrition as defined by SGA}

The association between CGA measures collected on the interRAI-AC and undernutrition (SGA) were investigated. All variables relating to physical function and Activities of Daily Living (ADL) on the interRAIAC are categorical and rated on a scale of 0 to 6 , with 0 meaning "independent", 1 independent with set up help and 2-6 being grades of modified independence or impairment. For the purposes of this analysis, these interRAI-AC variables were categorised into either "independent" or "not independent". Variables relating to fatigue, pain and sadness/depression are rated as zero (none) with categories above one representing various levels of severity or timing (for example, presence in the past 3 days to 24 hours). The variable for pressure ulcer 
Table 1

Characteristics of the sample

\begin{tabular}{|c|c|c|c|c|}
\hline VARIABLE & No SGA completed $(n=446)$ & SGA A $(n=62)$ & SGA B+C $(n=49)$ & P-value \\
\hline Age (years) ${ }^{*}$ & $81.5(7.0)$ & $83.3(6.2)$ & $84.1(5.1)$ & 0.437 \\
\hline Gender $(\mathrm{M} / \mathrm{F}) \dagger$ & $192 / 254$ & $24 / 38$ & $26 / 19$ & 0.148 \\
\hline Marital status $(\mathrm{N}, \%)+$ & & & & 0.346 \\
\hline - Never married & $28(6.3)$ & $4(6.5)$ & $3(6.7)$ & \\
\hline - Married & $169(37.9)$ & $26(41.9)$ & $16(35.6)$ & \\
\hline - Partner/significant other & $199(44.6)$ & $3(4.8)$ & $0(0)$ & \\
\hline - Widowed & $4(0.9)$ & $28(45.2)$ & $24(53.3)$ & \\
\hline - Divorced & $43(9.6)$ & $1(1.6)$ & $2(4.4)$ & \\
\hline - Missing & $3(0.7)$ & NA & NA & \\
\hline Country of birth $(\mathrm{N}, \%)+$ & & & & 0.798 \\
\hline - Australia & $227(50.9)$ & $16(25.8)$ & $26(57.8)$ & \\
\hline - Other & $110(24.7)$ & $11(17.7)$ & $19(42.2)$ & \\
\hline - Missing & $109(24.4)$ & $35(56.5)$ & $0(0)$ & \\
\hline Living arrangement $(\mathrm{N}, \%)+$ & & & & 0.280 \\
\hline - Alone & $163(36.5)$ & $21(33.9)$ & $16(35.6)$ & \\
\hline - With spouse/partner & $120(26.9)$ & $21(33.9)$ & $9(20.0)$ & \\
\hline - With spouse/partner/ others & $26(5.8)$ & $1(1.6)$ & $3(6.7)$ & \\
\hline - With child & $53(11.9)$ & $8(12.9)$ & $6(13.3)$ & \\
\hline - With sibling & $4(0.9)$ & $1(1.6)$ & $2(4.4)$ & \\
\hline - With other relatives & $3(0.7)$ & $2(3.2)$ & $0(0)$ & \\
\hline - With non-relatives & $63(14.1)$ & $5(8.1)$ & $9(20.0)$ & \\
\hline - Missing & $14(3.1)$ & $3(4.8)$ & $0(0)$ & \\
\hline Residence/ transition status $(\mathrm{N}, \%) \dagger$ & & & & 0.080 \\
\hline - Private residence - owned & $283(64.3)$ & $42(67.7)$ & $33(73.3)$ & \\
\hline - Private residence - rented & $35(8.0)$ & $6(9.7)$ & $2(4.4)$ & \\
\hline - Private residents - public rental & $32(7.3)$ & $2(3.2)$ & $1(2.2)$ & \\
\hline - Independent living in retirement village & $25(5.7)$ & $5(8.1)$ & $1(2.2)$ & \\
\hline - Short term crisis / emergency/ transitional accommodation & $4(0.9)$ & $1(1.6)$ & $1(2.2)$ & \\
\hline - RAC - low care & $23(5.2)$ & $2(3.2)$ & $1(2.2)$ & \\
\hline - RAC - high care & $24(5.5)$ & $1(1.6)$ & $1(2.2)$ & \\
\hline - Hospice/palliative care & $3(0.7)$ & $3(4.8)$ & $5(11.1)$ & \\
\hline - Acute care hospital & $11(2.5)$ & $0(0)$ & $0(0)$ & \\
\hline Time since last hospital stay & & & & 0.738 \\
\hline - no hospitalization within 90 days & $288(64.5)$ & $38(61.3)$ & $25(55.6)$ & \\
\hline - 31 to 90 days ago & $62(13.9)$ & $9(14.5)$ & $10(22.2)$ & \\
\hline - 15 to 30 days ago & $51(11.4)$ & $6(9.7)$ & $5(11.1)$ & \\
\hline - within 8 to 14 days & $25(5.6)$ & $4(6.5)$ & $1(2.2)$ & \\
\hline - in the last 7 days & $20(4.4)$ & $5(8.1)$ & $4(8.8)$ & \\
\hline Length of stay (days)* & $6(1-62)$ & $10(2-99)$ & $10(4-97)$ & 0.309 \\
\hline \multicolumn{5}{|l|}{ Polypharmacy (>6 medications) } \\
\hline - no & $230(51.6)$ & $45(72.5)$ & $20(40.8)$ & \\
\hline - yes & $216(48.4)$ & $17(27.4)$ & $29(59.1)$ & 0.170 \\
\hline \multicolumn{5}{|l|}{ Multi-morbidity ( $>3$ comorbidities) } \\
\hline- no & $244(54.7)$ & $48(77.4)$ & $26(53.1)$ & \\
\hline - yes & $202(45.3)$ & $14(22.6)$ & $23(46.9)$ & 0.372 \\
\hline Body mass index $\left(\mathrm{kg} / \mathrm{m}^{2}\right)^{*}$ & $24.0(5.3)$ & $24.0(6.2)$ & $22.0(5.1)$ & 0.143 \\
\hline
\end{tabular}

${ }^{*}$ Mean (SD) or median (range); +ANOVA, Kruskal Wallace or Chi-square test 
Table 2

CGA measures associated with undernutrition measured by SGA

\begin{tabular}{|c|c|c|c|c|}
\hline \multicolumn{2}{|c|}{ Variable } & \multirow{2}{*}{$\frac{\mathbf{N}}{111}$} & \multirow{2}{*}{$\begin{array}{l}\text { Chi-square statistic } \\
20.6\end{array}$} & \multirow{2}{*}{$\frac{\text { P value }}{0.000}$} \\
\hline 1. & Admission - activities of daily living (ADL): eating & & & \\
\hline 2. & Admission - short term memory / recall & 110 & 18.3 & 0.000 \\
\hline 4. & Admission - ADL: personal hygiene & 111 & 13.2 & 0.000 \\
\hline 5. & Weight loss $5 \%$ in last 30 days; $10 \%$ or more in last 180 days & 107 & 12.9 & 0.000 \\
\hline 8. & Admission - mode of nutritional intake & 111 & 10.2 & 0.001 \\
\hline 9. & Admission - ability to understand others & 111 & 7.8 & 0.005 \\
\hline 10. & Admission - fatigue & 111 & 7.5 & 0.006 \\
\hline 11. & Admission - little interest/pleasure in things enjoyed normally & 111 & 6.2 & 0.013 \\
\hline 12. & $\mathrm{BMI}<23 \mathrm{~kg} / \mathrm{m}^{2}$ & 88 & 4.8 & 0.029 \\
\hline
\end{tabular}

Table 3

Stepwise, backward logistic regression of the association between geriatric syndromes collected by the interRAI-AC at admission with undernutrition as measured by SGA

\begin{tabular}{|c|c|c|c|c|c|c|}
\hline Independent variables & Sample size & $\%$ undernutrition & Crude $\mathrm{OR}^{*}$ & Adjusted OR† & 95\% CI¥ & P-value \\
\hline \multicolumn{7}{|l|}{ Mode of nutritional intake } \\
\hline - Normal & 86 & 36 & 1.000 & 1.000 & Referent & \\
\hline - Modified or tube feeding & 25 & 72 & 4.562 & 3.756 & $0.813-17.364$ & 0.090 \\
\hline \multicolumn{7}{|l|}{ Feeling sad/depressed } \\
\hline - No & 75 & 33 & 1.000 & 1.000 & Referent & \\
\hline - Yes & 36 & 67 & 4.000 & 3.494 & $1.124-10.864$ & 0.031 \\
\hline \multicolumn{7}{|l|}{ Short term memory recall } \\
\hline - Not impaired & 53 & 23 & 1.000 & 1.000 & Referent & \\
\hline - Impaired & 57 & 63 & 5.857 & 3.325 & $1.152-9.594$ & 0.026 \\
\hline \multicolumn{7}{|c|}{$\begin{array}{l}\text { Weight loss of } 5 \% \text { in last } 30 \text { days or } 10 \% \\
\text { or more in last } 180 \text { days }\end{array}$} \\
\hline - No & 68 & 31 & 1.000 & 1.000 & Referent & \\
\hline - Yes & 39 & 67 & 4.476 & 2.877 & $0.983-8.416$ & 0.054 \\
\hline \multicolumn{7}{|l|}{ Fatigue } \\
\hline$-\mathrm{No}$ & 28 & 21 & 1.000 & 1.000 & Referent & \\
\hline - Yes & 80 & 51 & 3.855 & 3.494 & $1.414-43.205$ & 0.018 \\
\hline Model significance (Chi-sq) & 32.910 & 0.000 & & & & \\
\hline
\end{tabular}

Table 4

The performance of interRAI-AC undernutrition screener algorithm in patients with undernutrition

\begin{tabular}{|c|c|c|}
\hline \multirow{2}{*}{$\begin{array}{l}\text { Diagnosis according to interRAI-AC undernu- } \\
\text { trition screener algorithm }\end{array}$} & \multicolumn{2}{|c|}{ Dietitian diagnosis of malnutrition using the Subjective Global Assessment ${ }^{*}, \uparrow, \neq, \S,||,\{, \#$} \\
\hline & Undernutrition & Not undernutrition \\
\hline Positive diagnosis & 41 & 28 \\
\hline Negative diagnosis & 6 & 32 \\
\hline
\end{tabular}


severity is rated as zero (no pressure ulcer) with 1-4 being increasing levels of severity. Frequency analyses for these variables organised by SGA groupings (A, B or $C$ ) were examined to inform variable organisation for the analysis. Based on the small sample sizes (i.e. less than 10 cases) for each severity grading for fatigue, pain, sadness/depression and pressure ulcer, it was decided to dichotomise each of these variables to either "absent" or "present". Percentage weight loss of 5\% in last 30 days or $10 \%$ in last 180 days is categorised as either "yes" or "no". Chi-square tests presented in Table 2 show which items on the interRAI-AC were significantly associated with the presence of undernutrition according to SGA.

\section{Regression analyses}

Binary logistic regression with backward wald covariate search models were used to establish which variables collected by the interRAI-AC were having significant independent associations with undernutrition. Analyses for variables collected at admission are presented in Table 3. The final, most parsimonious model is presented.

All of the variables included in Table 3 had statistically significant crude odds ratios. Impairment in short term memory was associated with the largest magnitude increase in crude odds for undernutrition on admission (almost six-fold). When all variables were included in the multivariable logistic regression model, feeling sad or depressed, impairment in short term memory and the presence of fatigue were the most significant interRAIAC items associated with undernutrition on admission. Items relating to eating as an $\mathrm{ADL}$, cognitive skills for daily decision making, personal hygiene as an ADL, walking as an ADL, ability to understand others, interest and pleasure in things enjoyed normally and BMI were not statistically significant in the parsimonious regression model.

\section{Predictors of undernutrition at admission}

Compared to people with no reported fatigue on admission, those with fatigue had roughly three and a half times higher odds of being undernourished. Compared to those who were eating standard texture diets at admission, the odds of undernutrition in people who were not eating standard texture diets was roughly four and a half times higher. Compared to patients who had no short term memory problems on admission, those with short term memory impairment were roughly three times more likely to be undernourished. Patients who had experienced recent weight loss (5\% in 30 days; $10 \%$ in 180 days) were around four and a half times more likely to be undernourished, compared to those who did not have recent weight loss.

\section{Decision tree analysis}

Decision tree analyses were performed using significant independent variables from the logistic regression analysis. In model one, short term memory recalling ability and depression were able to separate the whole patient population into three groups in relation to undernutrition. In model two, short term memory recalling ability and recent weight loss (5\% or more in the last 30 days or $10 \%$ or more in the last 180 days) separated the population into three subgroups in relation to nutritional status (See figure 1).

\section{Figure 1}

Decision tree analysis showing percentage weight loss and short-term memory recall items present in the interRAI-AC grouping patients into three groups

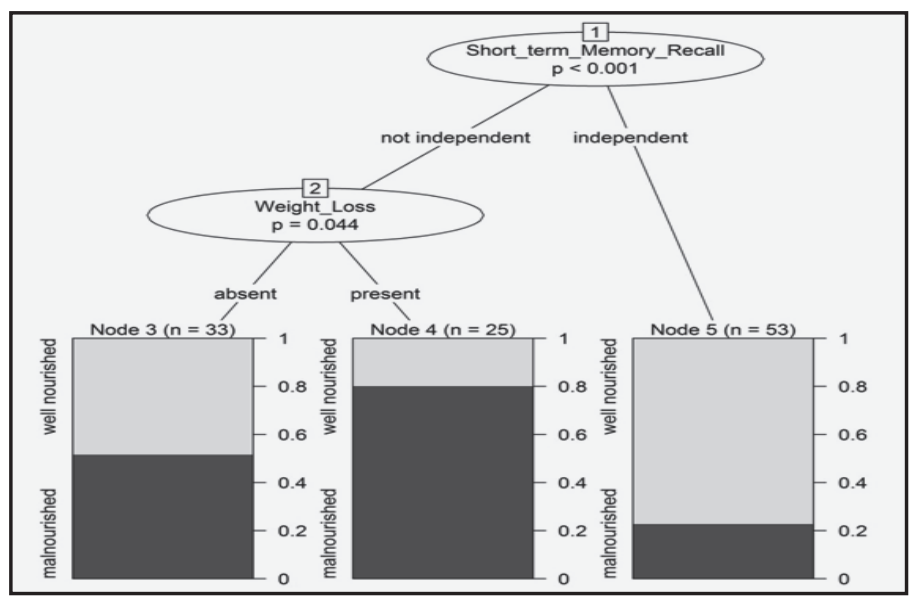

\section{Receiver Operating Characteristic (ROC) Curve analysis}

The results from the decision tree analysis were assessed via ROC curve analysis to evaluate their sensitivity and specificity for predicting the presence (or absence) of undernutrition on admission. Decision tree analysis one, including the variables short term memory recall and sad/depressed and hopeless, produced an area under the curve (AUC) of 72.8\% (95\% CI: 65\%-80.6\%), while decision tree analysis two, including the variables short term memory recall and percentage weight loss, was marginally better, returning an AUC of $74.8 \%$ (95\% CI: $65.9 \%-83.6 \%$ ).

\section{Development of the undernutrition screener algorithm}

The aim of the development of the interRAI-AC undernutrition algorithm was to maximise sensitivity to facilitate thorough identification of patients who were undernourished. We utilised the combination of items with the highest AUC estimate. The algorithm 
developed included a combination of recent weight loss $(5 \%$ or more in the last 30 days or $10 \%$ or more in the last 180 days) and short term memory recalling ability. Of the 49 patients diagnosed by dietitians as being undernourished, 2 had missing values on the interRAI-AC undernutrition screener due to 2 missing values for the recent weight loss component. Similarly, 2 of the 62 patients diagnosed by dietitians as not being undernourished had missing values on the interRAI-AC undernutrition screener for this variable. Therefore, of the 47 patients diagnosed by dietitians as undernourished, 41 were correctly rated as undernourished by the interRAI-AC undernutrition screener algorithm, yielding $87 \%$ sensitivity, $53 \%$ specificity, positive and negative predictive values of $59 \%$ and $84 \%$.

\section{Discussion}

This study revealed three significant findings: (i) the interRAI-AC incorporates measures that coincide with internationally-recognised clinical definitions of undernutrition, i.e. the International Statistical Classification of Diseases and Related Health Problems Australian Modification, including: BMI, percentage weight loss and evidence of suboptimal intake; however, not all of these items were statistically associated with the presence of undernutrition as measured by the SGA in this study; (ii) items examining percentage weight loss over time, self-reported fatigue, depression and short term memory on admission contribute significantly to the prediction of undernutrition in older adults in acute care; (iii) the combination of self-reported short term memory recall and percentage weight loss provides the most statistically robust screener for undernutrition within the interRAI-AC at present.

A large proportion of nutrition screening practices used in geriatric settings rely on monitoring weight loss or BMI, or combinations of both (15). BMI $<18.5 \mathrm{~kg} /$ $\mathrm{m}^{2}$ or unintentional weight loss $(5-9 \%$ or $\geq 10 \%)$ with "evidence of suboptimal intake resulting in mild loss of subcutaneous fat and/or mild muscle wasting" are used to define undernutrition codes in the International Statistical Classification of Diseases and Related Health Problems Australian Modification (ICD-10-AM). Undernutrition is coded in the hospital medical record based on these criteria and is linked to increased health care reimbursements. Our study revealed unintentional weight loss $(5 \%$ or more in the last 30 days or $10 \%$ or more in the last 180 days) as a significant predictor of undernutrition at admission in older adults; however BMI was not statistically significant, despite the testing of several different cut-offs, including BMI $<18.5 \mathrm{~kg} /$ $\mathrm{m}^{2},<23 \mathrm{~kg} / \mathrm{m}^{2}$ and $<25 \mathrm{~kg} / \mathrm{m}^{2}$. Percentage weight loss over time is a component of several nutritional screening and assessment tools (15). It is more sensitive as an indicator of subcutaneous fat loss and muscle wasting than weight alone, as spontaneous fluid shifts in hospital may cause inaccurate results. BMI does not adjust for the proportion of lean and fat tissue, and has been shown to underestimate undernutrition (16). There is also significant potential for error in the measurement (or reporting of) height and weight in hospital, reducing confidence in estimates relying on this information. It is therefore advantageous that one of the potential nutrition screeners we have derived from the interRAI-AC utilises percentage weight loss rather than BMI.

Poor short term recalling ability was a component of the interRAI-AC screener for undernutrition developed in this study. Cognitive impairment has been shown to increase the risk of undernutrition in older adults living in their own homes, and in nursing homes (17), and is associated with higher all-cause mortality in hospital (18); however, no previous nutrition screeners have incorporated such an item. In the MNA-SF, cognitive status is briefly mentioned within "neuropsychological problems", but focuses on the presence of "dementia or depression" rather than short term memory issues. Selfreported sadness, depression or feelings of hopelessness contributed to the prediction of undernutrition on admission in this study, yet this type of item (depression/ hopelessness only) is also absent from current nutrition screening tools. Recent studies have confirmed the importance of considering the presence of cognitive impairment and depression in the identification of undernutrition in older patients (19), therefore we consider our screener adds significant novelty to the field.

Fatigue on admission was significantly associated with admission undernutrition in our study. Undernutrition is recognised as a consequence of fatigue (20). Fatigue is also associated with acute undernutrition and poor food intake in hospital, as exhaustion prevents the ability to eat independently and to chew and swallow for the time it takes to finish a meal (21). Although it was not determined to be the most statistically robust item in our decision tree analysis, inclusion of the interRAI-AC item about fatigue in further validation studies with larger sample sizes is important, as this variable may assist in distinguishing between acute and chronic undernutrition on admission and this is of high clinical significance. No nutritional screening tools currently measure fatigue on admission therefore our work contributes novelty to the field.

Analysis of AUC indicated the screeners developed here would accurately predict the presence of undernutrition around $75 \%$ of the time. This is comparable to the predictive ability of other nutrition screening tools validated against the SGA (22-25). The Malnutrition Screening Tool (MST) is the most common nutrition screener used in Australian hospitals and has demonstrated a higher sensitivity (90-94\%) and specificity $(85-89 \%)$ against the SGA in hospital patients aged over 65 years $(26,27)$, with lower sensitivity and specificity in a larger sample of older adults in residential aged care $(n=258)(83 \%$ sensitivity; $64 \%$ specificity) $(28)$. The 
MNA-SF has very high demonstrated sensitivity (100\%) against the SGA in elderly hospital patients aged 65 years and older; however, the specificity is lower (52.8\%) (27). This is attributed to its more global assessment approach, as it includes a section for cognitive assessment and patients receive higher scores if they are in hospital. These statistical parameters and the cognitive component are similar to our new interRAI-AC nutrition screener, although our screener contains only two items compared to the MNA-SF's 7 items. Despite these instruments being over-inclusive, they increase the likelihood of treatment of patients who are undernourished and the potential for prevention of malnutrition in the false positives, which increases in relevance as length of stay increases.

The interRAI-AC items for short term memory recall ability, percentage weight loss and feelings of sadness, depression or hopelessness have demonstrated good to excellent inter-rater reliability with kappas of 0.6 (95\% CI: 0.44-0.75), 0.74 (95\% CI: $0.88-0.91)$ and 0.92 (95\% CI: 0.85 - 1.00) respectively (29). Inter-rater reliability for other nutrition screening tools has not been widely studied; however our screener is comparable to the MST, which has excellent demonstrated inter-rater reliability of kappa $0.88(22)$.

Our study showed that $111 / 557$ patients had received a nutritional assessment (20 per cent) as a result of standard practice at the hospital. Of that 20 per cent, around 44 per cent were undernourished. It is possible that at least 44 per cent of the remaining patients who had not received a nutritional assessment were also malnourished; however, they would not have received any targeted interventions, increasing their risk of nutritional decline in hospital and potentially, postdischarge. The incorporation of a malnutrition screener into CGA which is standard practice, has the potential to improve the identification of risk of malnutrition on admission in older adults, thereby improving referral rates to dietitians and other practitioners for welltargeted interventions.

\section{Limitations}

A limitation is that the data examined in this study was analysed retrospectively. The reliance on already completed SGA data meant that the study included patients already identified as "at risk" of undernutrition by standard screening practices at the hospital; however, this screening data was unavailable. The study was also limited by the relatively small proportion of nutritional assessments conducted as standard practice in this group of patients $(n=111 / 557)$. Despite this, the similar trends identified using the larger dataset $(n=557)$ in relation to the demographic and community service utilisation of patients, and the gender differences identified using the larger dataset $(\mathrm{n}=557)$, was a major strength of the study. The SGAs were completed by a range of dietitians and there is no inter-rater reliability data available; however, this tool has demonstrated good inter-rater reliability in previous work (6).

\section{Conclusions}

This study has identified several measures within the interRAI-AC that can be used as part of a screener for undernutrition in acute hospital patients aged 70 years or older. The combination of short term memory recall and percentage weight loss provides the most statistically robust screener for undernutrition within the interRAI-AC at present. This is the first study to quantify the potential for CGA to contribute to screening for undernutrition in acute care and demonstrates the significant potential for, and advantages of, multidisciplinary collaboration in the development and refinement of assessment tools in geriatric medicine.

The inclusion of measures encompassing cognition and/or depression alongside percentage weight loss in a screener for undernutrition is valuable, as it provides context around the diagnosis of undernutrition. The majority of nutrition screening and assessment tools focus on measures of weight and suboptimal food intake, but do not provide measures of potential explanations for these results, apart from poor appetite (8). The advantage of a nutrition screener with internationally-recognised clinical indicators (ICD-10-AM) in addition to contextual items incorporated within CGA, is that results can be interpreted alongside substantial clinical information about geriatric syndromes, demographic details, living arrangements and prior community services. This assists in determining the most effective and targeted intervention to either prevent nutritional decline or reduce the extent of undernutrition present while in hospital, in addition to planning appropriate care and services upon discharge.

Ethical Standards: Princess Alexandra Hospital HREC (2004/079; 2008/130) UQ MREC (2008001564)

Conflicts of Interest: NIL - The authors have no conflict of interest.

\section{References}

1. Stuck AE, Siu AL, Wieland GD, Adams J, Rubenstein LZ. Comprehensive geriatric assessment: a meta-analysis of controlled trials. Lancet. 1993;342(8878):1032-6.

2. Aitken PD, Rodgers H, French JM, Bates D, James OFW. General Medical or Geriatric Unit Care for Acute Stroke? A Controlled Trial. Age and Ageing. 1993;22(suppl 2):P4.

3. Isabel T. D. Correia M, Waitzberg DL. The impact of malnutrition on morbidity, mortality, length of hospital stay and costs evaluated through a multivariate model analysis. Clinical Nutrition. 2003;22(3):235-9.

4. Ellis G, Langhorne P. Comprehensive geriatric assessment for older hospital patients. British Medical Bulletin. 2005;71(1):45-59.

5. McWhirter JP. The incidence and recognition of malnutrition in hospital. British Medical Journal. 1994;308(6934):945-8.

6. Detsky A, McLaughlin, Baker J, Johnston N, Whittaker S, Mendelson R, et al. What is subjective global assessment of nutritional status? Journal of Parenteral and Enteral Nutrition. 1987;11(1):8-13.

7. Ottery FD. Patient-Generated Subjective Global Assessment. In: McCallum PD, Polisena CG, editors. The Clinical Guide to Oncology Nutrition. Chicago: The American Dietetic Association; 2000. p. 11-23.

8. Ferguson M, Capra S, Bauer J, Banks M. Development of a valid and reliable 
malnutrition screening tool for adult acute hospital patients. Nutrition. 1999;15(6):458-64.

9. Christensson L, Unosson M, Ek A-C. Evaluation of nutritional assessment techniques in elderly people newly admitted to municipal care. Eur J Clin Nutr. 2002;56(9):810-8.

10. Lakhan P, Jones M, Wilson A, Courtney M, Hirdes J, Gray LC. A Prospective Cohort Study of Geriatric Syndromes Among Older Medical Patients Admitted to Acute Care Hospitals. Journal of the American Geriatrics Society. 2011;59(11):2001-8.

11. interRAI. interRAI International Website: We-Create Enterprise Systems; 2008 [updated 13/02/2008; cited 200825 Feb]. Available from: http://www. interrai.org/section/view/.

12. Gray LC, Bernabei R, Berg K, Finne-Soveri H, Fries BE, Hirdes JP, et al Standardizing Assessment of Elderly People in Acute Care: The interRAI Acute Care Instrument. Journal of the American Geriatrics Society. 2008;56(3):536-41.

13. Hirdes J, Ljunggren G, Morris J, Frijters D, Finne Soveri H, Gray L, et al. Reliability of the interRAI suite of assessment instruments: a 12-country study of an integrated health information system. BMC Health Services Research. 2008;8(1):277.

14. Jonsson PV, Finne-Soveri H, Jensdottir AB, Ljunggren G, Bucht G, Grue EV et al. Co-morbidity and functional limitation in older patients underreported in medical records in Nordic Acute Care Hospitals when compared with the MDS-AC instrument. Age Ageing. 2006 Jul;35(4):434-8.

15. Evidence based practice guidelines for the nutritional management of malnutrition in adult patients across the continuum of care. Nutrition \& Dietetics. 2009;66:S1-S34.

16. Beck AM, Ovesen L. At which body mass index and degree of weight loss should hospitalized elderly patients be considered at nutritional risk? Clinical Nutrition. 1998;17(5):195-8.

17. Fagerström C, Palmqvist R, Carlsson J, Hellström Y. Malnutrition and cognitive impairment among people 60 years of age and above living in regular housing and in special housing in Sweden: A population-based cohort study. International journal of nursing studies. 2011;48(7):863-71.

18. Farid K, Zhang Y, Bachelier D, Gilson P, Teixeira A, Safar ME, et al. Cognitive impairment and malnutrition, predictors of all-cause mortality in hospitalized elderly subjects with cardiovascular disease. Archives of Cardiovascular Diseases. 2013;106(4):188-95.
19. van Bokhorst-de van der Schueren MAE, Lonterman-Monasch S, de Vries OJ, Danner SA, Kramer MHH, Muller M. Prevalence and determinants for malnutrition in geriatric outpatients. Clinical nutrition. 2013;32:1007-1011.

20. Tennant KF, Takacs SE, Gau JT, Clark BC, Russ DW. A Preliminary Study of Symptomatic Fatigue in Rural Older Adults. Aging Clin Exp Res. 2011;24(4):324-30.

21. Tierney AJ. Undernutrition and elderly hospital patients: a review. Journal of Advanced Nursing. 1996;23(2):228-36

22. Ferguson M, Capra S, Bauer J, Banks M. Development of a valid and reliable malnutrition screening tool for adult acute hospital patients. Nutrition. 1999;15(6):458-64.

23. Kondrup J, Rasmussen HH, Hamberg OLE, Stanga Z. Nutritional risk screening (NRS 2002): a new method based on an analysis of controlled clinical trials. Clinical nutrition. 2003;22(3):321-36.

24. Kruizenga H, Seidell J, de Vet H, Wierdsma N, van Bokhorst-de van der Schueren M. Development and validation of a hospital screening tool for malnutrition: the Short Nutritional Assessment Questionnaire (SNAQ). Clin Nutr. 2005;24:75-82.

25. Rubenstein LZ, Harker JO, Salvà A, Guigoz Y Vellas B. Screening for Undernutrition in Geriatric Practice: Developing the Short-Form MiniNutritional Assessment (MNA-SF). The Journals of Gerontology Series A: Biological Sciences and Medical Sciences. 2001;56(6):M366-M72.

26. Wu M-L, Courtney MD, Shortridge-Baggett LM, Finlayson K, Isenring EA. Validity of the Malnutrition Screening Tool for Older Adults at High Risk of Hospital Readmission. J Gerontol Nurs. 2012;38(6):38-45.

27. Young AM, Kidston S, Banks MD, Mudge AM, Isenring EA. Malnutrition screening tools: Comparison against two validated nutrition assessmen methods in older medical inpatients. Nutrition. 2013;29(1):101-6

28. Isenring EA, Bauer JD, Banks M, Gaskill D. The Malnutrition Screening Tool is a useful tool for identifying malnutrition risk in residential aged care. Journal of Human Nutrition and Dietetics. 2009;22(6):545-50.

29. Wellens NIH, Van Lancker A, Flamaing J, Gray L, Moons P, Verbeke G, et al. Interrater reliability of the interRAI Acute Care (interRAI AC). Archives of gerontology and geriatrics. 2012;55(1):165-72.

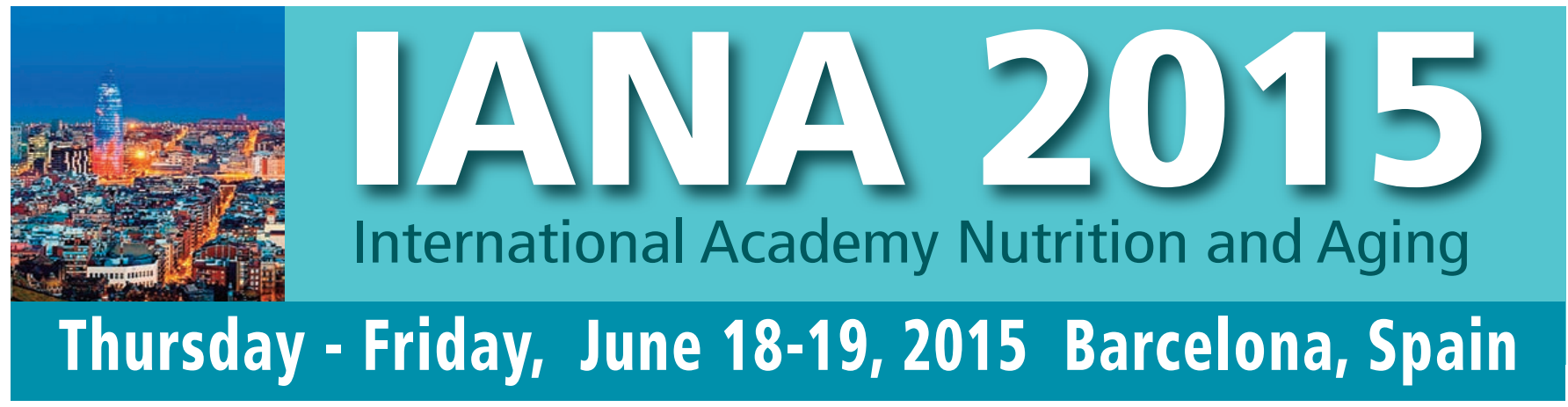

Nutrition and cognitive decline Alzheimer $\bullet$ Nutritional Assessment, MNA $\bullet$ Dietary intake and data sharing $\bullet$ Dietary recommendations for healthy aging $\bullet$ New technologies to assess nutritional status and diet $\bullet$ Aging Gut
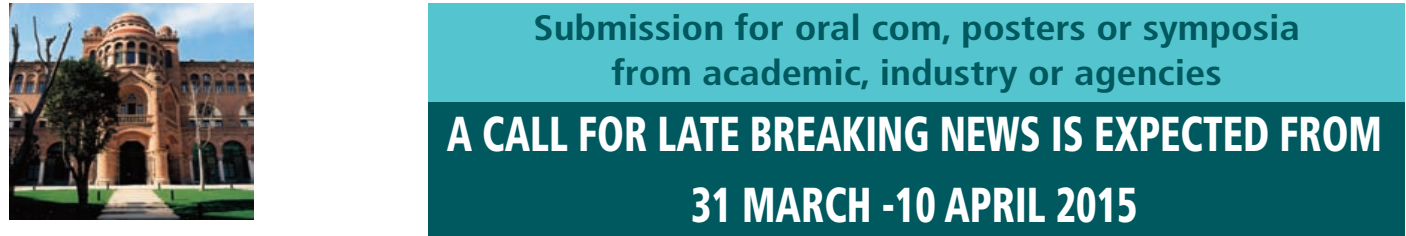

Meeting Place:

Hospital de la Santa Creu i Sant Pau

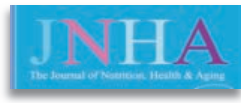

Proceedings

of the conference

will be published in the JNHA the Journal of Nutrition

Health and Aging 